\title{
Impact of 3D Antenna Radiation Patterns on TDOA-Based Wireless Localization of UAVs
}

\author{
Priyanka Sinha, Yavuz Yapici, and Ismail Guvenc \\ Department of Electrical and Computer Engineering, North Carolina State University, Raleigh, NC \\ Email: $\{$ psinha2, yyapici, iguvenc\}@ncsu.edu
}

\begin{abstract}
Next big commercial applications of drones require to fly the drone beyond the visual line of sight (BVLOS). This inevitable ability to fly BVLOS will also necessitate the ability to keep track of the drone's location, in order to ensure successful completion of the intended service. In this context, we explore the fundamental limits of 3D localization of drones in conjunction with the effects of the 3D antenna radiation patterns. Although localization of drone/unmanned aerial vehicle (UAV) is a well-studied topic in the literature, its relationship to the antenna effects remains mostly unexplored. In this paper, we investigate the impact of antenna radiation pattern on the accuracy of time-difference-of-arrival (TDOA)-based localization of the UAV. Specifically, we consider a scenario where a fixed number of radio-frequency (RF) sensors, placed at some known locations on the ground, collect the TDOA measurements from the signals transmitted from the UAV, and estimate the location of the UAV from these observations. In order to study the impact of the antenna effects on the fundamental limits of the TDOA-based positioning scheme, we develop a simple analytical model to approximate the total antenna gains experienced by an air-to-ground (A2G) link, for various orientations of the antennas. We then derive the Cramer-Rao lower bound for the TDOA based localization scheme, for all three combinations of the transmit and the receive antenna orientations: vertical-vertical (VV), horizontal-horizontal (HH), and vertical-horizontal (VH).
\end{abstract}

Index Terms-Antenna effects, cellular positioning, Cramer-Rao lower bound (CRLB), drone-localization, TDOA based localization, unauthorized drone detection.

\section{INTRODUCTION}

Drone localization has a wide range of applications in military, commercial, government, and recreational scenarios. Depending on the context, the drone may or may not participate in the localization process, either because the drone communication system might not be designed for positioning purposes, or it may be a non-cooperating and potentially malicious drone [1]-[3]. There has been some recent studies in the literature to use the radio frequency (RF) signals from drones for the purpose of detection, classification, localization, and tracking of drones, regardless of whether the drone is cooperating or not [2]-[5].

There are several parameters associated with an RF signal that can be measured and used to localize of the source of the signal, such as the received signal strength (RSS), angle-of-arrival (AOA), time-of-arrival (TOA), and time-difference-of-arrival (TDOA) [6]. Due to the simplicity of implementation, localization schemes involving RSS, TOA, and TDOA are more common as compared to the AOA methods, and the time based methods are usually preferred over the RSS based methods due to the higher degree of accuracy they provide. Between the TOA and the TDOA based approaches, TDOA is more suitable to a wider range of general

This work has been supported in part by NASA under the Federal Award number NX17AJ94A.

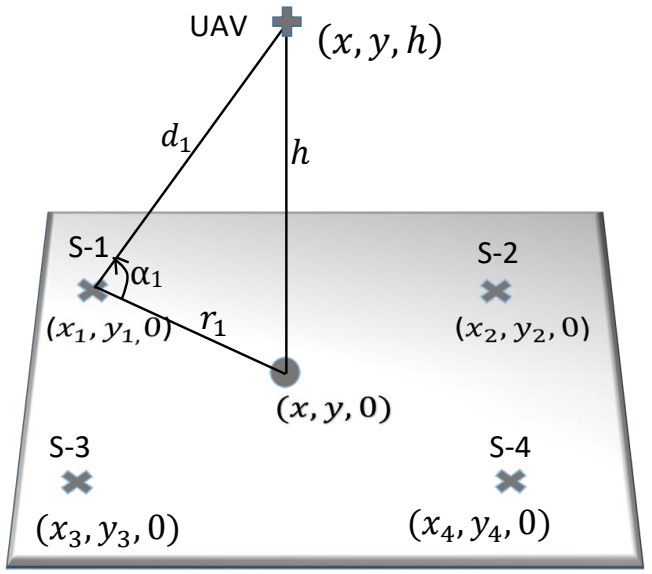

Fig. 1: Reference sensors with fixed locations, that collect TOA measurements for drone localization.

applications, as it does not require the source clock to be synchronized with that of the RF sensor.

Due to their advantages, the TDOA based localization schemes have been studied extensively in the literature. In [7], the authors provide a survey of the existing time of arrival (TOA) based algorithms, provide associated Cramer-Rao lower bound (CRLB) for different approaches, and review non-line-of-sight (NLOS) mitigation techniques in the literature. In [8], the authors study the fundamental limits of TOA-based localization scheme for wireless sensor networks. In [9], TDOA-based method is introduced for direct position estimation in a 2D plane and the associated CRLB are derived. In spite of the large volume of the existing literature, a study of the TDOA based localization that takes the effect of antenna radiation patterns into account is lacking in the literature to our best knowledge. The effect of the antenna patterns on the air-to-ground (A2G) channels is investigated in [10], and the impact of the antenna patterns on the received signal strength at the ground units is demonstrated.

In this paper, we consider a simple antenna model as used in [10], and we study the impact of the antenna alignment on the fundamental limits of the 3D localization of a UAV. To the best of our knowledge, such work is not a part of the literature yet. The main contributions of our work are as follows: 1) a simple yet effective analytical model is introduced to approximate the antenna gains in the $A 2 G$ link for the different orientations of the transmit and the receive antennas; 2) closed form expressions are derived demonstrating the impact of antenna gains on the variance of the TDOA measurement noise, assuming the availability of Line-of-sight (LOS) A2G links; 3) the CRLB on the TDOA based localization is derived; and finally 4) the performance of the localization scheme for the different antenna patterns are characterized, and 
computer simulations are used to evaluate localization coverage and accuracy metrics for various antenna configurations.

\section{SYSTEM MODEL}

\section{A. Extraction of the TOA from RF Signals}

The TDOA measurement between a pair of time synchronized RF sensors is obtained by calculating the difference between the TOA estimates measured at each of these sensors. Subtracting one TOA estimate from another eliminates the dependence of the estimated distance between the UAV and the sensor, on the unknown time of transmission at the UAV. Assuming that an efficient maximum-likelihood (ML) estimator is used at the sensors to obtain the TOA estimates, the TOA measurement noise at the sensors can be modelled as zero-mean Gaussian noise, due to the asymptotic normality property of ML estimators. We consider a system model where we have a fixed number of ground RF-sensors or fixed terminals (FTs) with known locations $\left(x_{i}, y_{i}\right)$, to collect the TOA measurements from the signals arriving from a UAV located at $(x, y, h)$. Fig. 1 illustrates such a model with four ground FTs, where it is desired to estimate the 3-D location coordinates $(x, y, h)$ of the UAV.

Let the 3D distance between the UAV and the $i^{\text {th }}$ sensor, $\mathrm{S}-i$, be denoted as $d_{i}$, and let the actual (measured) value of the TOA be denoted as $\tau_{i}\left(\hat{\tau}_{i}\right)$. Then the relationship among these quantities are given as:

$$
\begin{aligned}
d_{i} & =\sqrt{\left(x_{i}-x\right)^{2}+\left(y_{i}-y\right)^{2}+h^{2}}, \\
\tau_{i} & =\frac{d_{i}}{c}+t_{0}, \\
\hat{\tau}_{i} & =\tau_{i}+n_{i},
\end{aligned}
$$

where $\mathrm{n}_{1} \sim \mathcal{N}\left(0, \sigma_{i}^{2}\right)$, is the additive white Gaussian measurement noise, and $c$ is the speed of propagation. The TOA estimates in (3) are conventionally obtained from correlating receivers. We assume that each of the sensors intercepts the UAV signal within the time interval $(0, T)$, where the UAV signal is assumed to have a known waveform $s(t)$ that is transmitted for a known duration of $T_{s} \leq T$, starting from an unknown time $t_{0} \leq T$. As it is not unusual to have a dominant line-of-sight (LOS) path for A2G channels, the signal received at the $i^{\text {th }}$ sensor, in presence of additive white Gaussian noise (AWGN) can be given as:

$$
r_{i}(t)=A_{i} s\left(t-\tau_{i}\right)+n(t),
$$

where $\tau_{i}$ is the true value of the TOA at the $i^{\text {th }}$ BS, $A_{i}$ is the amplitude factor representing large scale fading, and $n(t)$ is the white Gaussian noise with zero mean and spectral density $\frac{N_{0}}{2}$. Please note that, due to the assumption of having a strong LOS component, we ignore the impact of Doppler spread, as the number of multipath components and the angular spread of the incoming waves are expected to decrease, which in turn reduces the Doppler spread [11]. It is desired to estimate the time of arrival, $\tau_{i}$ in (4), and for this continuous time model, the log-likelihood function [9] for the $i^{\text {th }}$ sensor is given as:

$$
l\left(\tau_{i}\right)=-\frac{1}{N_{0}} \int_{0}^{T}\left(r_{i}(t)-A_{i} s\left(t-\tau_{i}\right)\right)^{2} d t .
$$

Since we argue that the above ML estimator achieves asymptotic optimality in a LOS-dominated environment, we conclude that the covariance of the TOA estimates at all the sensors attains the CRLB, and is given as the inverse of the Fisher Information Matrix (FIM), $\boldsymbol{I}(\boldsymbol{\tau})$.

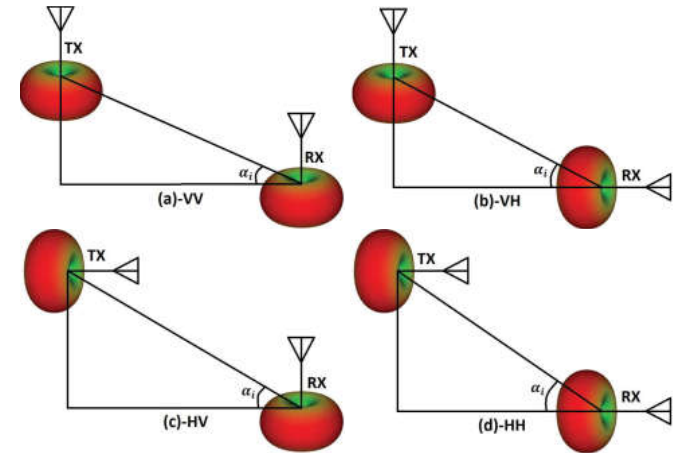

Fig. 2: Analytical model for the antenna gains considering vertical-vertical antenna configuration.

According to [12], the corresponding FIM is obtained as: $\boldsymbol{I}(\boldsymbol{\tau})=\frac{\partial \boldsymbol{r}^{T}(\tau)}{\partial \tau} \Sigma^{-1} \frac{\partial \boldsymbol{r}(\tau)}{\partial \tau}$, where $\boldsymbol{r}=\left[r_{1}, r_{2}, r_{3}, . . r_{N}\right]^{T}$ is the received signal vector and $\Sigma$ is the covariance of the AWGN at the sensors. Since we assume independent and identically distributed (iid) noise samples, $\boldsymbol{\Sigma}$ becomes a diagonal matrix $\sigma^{2} \boldsymbol{I}_{N}$, and inverse FIM is

$$
\boldsymbol{I}^{-1}(\boldsymbol{\tau})=\left(\begin{array}{cccc}
\sigma_{1}^{2} & 0 & \cdots & 0 \\
0 & \sigma_{2}^{2} & \cdots & 0 \\
\vdots & \vdots & \ddots & \vdots \\
0 & 0 & \cdots & \sigma_{N}^{2}
\end{array}\right)
$$

Due to the efficient estimator assumption, the TOA measurement noise at the $i^{\text {th }}$ sensor, in (3), becomes a zero-mean Gaussian random variable with variance $\sigma_{i}^{2}=$ $\left[\boldsymbol{I}^{-1}(\boldsymbol{\tau})\right]_{i i}$, which can also be written as [6]:

$$
\sigma_{i}=\frac{1}{2 \sqrt{2} \pi \sqrt{S N R_{i}} \beta}=\frac{k}{S N R_{i}},
$$

where $\beta$ is the effective drone signal bandwidth, and $S N R_{i}=\frac{A_{i}{ }^{2} E_{S}}{N_{0}}$, with $E_{s}$ representing the energy in the transmit signal $s(t)$. For the broader objective of this paper we summarize the impact of factors other than signal-to-noise ratio (SNR) on the TOA measurement noise, using a proportionality constant $k$.

\section{B. Analytical Model for Antenna Pattern}

In order to study the impact of the antenna radiation patterns on the TOA CRLB, we characterize the relationship between the total antenna gain experienced at the $i^{\text {th }}$ sensor, and the variance of the TOA measurement noise. In this paper, we use a simple antenna gain function to characterize the gain from dipole antenna radiation patterns [10]. In particular, we approximate the antenna gain as a sine/cosine function of the elevation angle $\left(\alpha_{i}\right)$ between the aerial transmitter (the UAV), and the receiver at the $i^{\text {th }}$ ground sensor depending on the orientation of the transmit and receive antennas. We consider 4 such antenna orientations, namely, the VV pattern, where both Tx and $\mathrm{Rx}$ antennas are oriented vertically, the $\mathrm{HH}$ pattern, where both the transmit and the receive antennas are oriented horizontally, and the $\mathrm{VH} / \mathrm{HV}$ pattern, where the transmit and the receive antennas are respectively, vertically and horizontally and vertically and horizontally oriented.

TABLE I: Total antenna gain for different antenna patterns

\begin{tabular}{|c||c|c|c|}
\hline & $\mathbf{V V}$ & $\mathbf{V H}$ & $\mathbf{H H}$ \\
\hline$G_{\mathrm{RX}}\left(\alpha_{i}\right)$ & $\cos \left(\alpha_{i}\right)$ & $\cos \left(\alpha_{i}\right)$ & $\sin \left(\alpha_{i}\right)$ \\
\hline$G_{\mathrm{TX}}\left(\alpha_{i}\right)$ & $\cos \left(\alpha_{i}\right)$ & $\sin \left(\alpha_{i}\right)$ & $\sin \left(\alpha_{i}\right)$ \\
\hline$G_{\mathrm{RX}}\left(\alpha_{i}\right) G_{\mathrm{TX}}\left(\alpha_{i}\right)$ & $\cos ^{2}\left(\alpha_{i}\right)$ & $0.5 \sin \left(2 \alpha_{i}\right)$ & $\sin ^{2}\left(\alpha_{i}\right)$ \\
\hline
\end{tabular}

Fig. 2(a) and Fig. 2(b) show the VV and the $\mathrm{HH}$ scenario for antenna radiation patterns, respectively, where 
the doughnut-like radiation pattern in the 3D plane are also illustrated. The $\mathrm{VH}$ and the $\mathrm{HV}$ patterns are identical in terms of the total antenna gain, and are illustrated in Fig. 2(c) and Fig. 2(d), respectively. The total antenna gain experienced at the receiver, $G_{\mathrm{TX}} G_{\mathrm{RX}}$, for the different scenarios are summarized in Table [].

As a result, we can represent the RSS at the $i^{\text {th }}$ ground receiver from an aerial transmitter as:

$$
P_{\mathrm{RX}}\left(\alpha_{i}\right)=P_{\mathrm{TX}} G_{\mathrm{TX}}\left(\alpha_{i}\right) G_{\mathrm{RX}}\left(\alpha_{i}\right)\left(\frac{\lambda}{4 \pi d_{i}}\right)^{\gamma},
$$

where $P_{\mathrm{TX}}$ is the transmitted signal power at the UAV, $G_{\mathrm{TX}}\left(\alpha_{i}\right) \leq 1$ is the transmitter antenna gain, $G_{\mathrm{RX}}\left(\alpha_{i}\right) \leq$ 1 is the receiver antenna gain, $\alpha_{i}$ and $d_{i}$ are the elevation angle and the 3-D distance between the transmitter and the receiver, respectively, $\lambda$ is the wavelength, and $\gamma$ is the path loss exponent, which is assumed as two in the rest of this paper due to LOS assumption. With help of (8), we can now represent the receive SNR as a function of the antenna gains:

$$
S N R_{i}=\frac{P_{\mathrm{RX}}\left(\alpha_{i}\right)}{\sigma_{\mathrm{n}}{ }^{2}}=\left(\frac{\lambda}{4 \pi}\right)^{2} \frac{P_{\mathrm{TX}} G_{\mathrm{TX}}\left(\alpha_{i}\right) G_{\mathrm{RX}}\left(\alpha_{i}\right)}{\sigma_{\mathrm{n}}{ }^{2} d_{i}{ }^{2}},
$$

where $\sigma_{\mathrm{n}}{ }^{2}$ is the variance of the thermal AWGN. Using (7), the relationship between the total antenna gain, and the variance of the TOA measurement noise at the $i^{\text {th }}$ sensor is:

$$
\sigma_{i}^{2}=\frac{k_{0} \sigma_{\mathrm{n}}{ }^{2} d_{i}{ }^{2}}{P_{\mathrm{TX}} G_{\mathrm{TX}}\left(\alpha_{i}\right) G_{\mathrm{RX}}\left(\alpha_{i}\right)},
$$

where the proportionality constant $k_{0}$ summarizes the impact of all factors other than the total antenna gain, AWGN noise variance and the 3-D distance between the UAV and the $i^{\text {th }}$ sensor, and is given as [13]: $k_{0}=\frac{2 E f^{2}}{\beta^{2} c^{2}}$, where $\beta$ is the bandwidth of the drone signal, $E$ is the energy in the drone signal at the UAV transmitter, $f$ is the center operating frequency of the ground sensors, and $c$ is the speed of propagation. Using the expression for the elevation angle $\alpha_{i}=\tan ^{-1}\left(\frac{h}{\sqrt{\left(x-x_{i}\right)^{2}+\left(y-y_{i}\right)^{2}}}\right)$, in (10), the relationship between the variance of the TOA measurement noise $\sigma_{i}^{2}$, and the unknown location of the UAV $(x, y, h)$, can be represented as follows:

$$
\begin{gathered}
{\sigma_{\mathrm{i}-\mathrm{Vv}}}^{2}=\frac{k_{0}{\sigma_{\mathrm{n}}}^{2}\left(\left(x-x_{i}\right)^{2}+\left(y-y_{i}\right)^{2}+h^{2}\right)^{2}}{\left(x-x_{i}\right)^{2}+\left(y-y_{i}\right)^{2}}, \\
{\sigma_{\mathrm{i}-\mathrm{HH}}}^{2}=\frac{k_{0}{\sigma_{\mathrm{n}}}^{2}\left(\left(x-x_{i}\right)^{2}+\left(y-y_{i}\right)^{2}+h^{2}\right)^{2}}{h^{2}}, \\
{\sigma_{\mathrm{i}-\mathrm{VH}}}^{2}=\frac{k_{0}{\sigma_{\mathrm{n}}}^{2}\left(\left(x-x_{i}\right)^{2}+\left(y-y_{i}\right)^{2}+h^{2}\right)^{2}}{h \sqrt{\left(x-x_{i}\right)^{2}+\left(y-y_{i}\right)^{2}},} \\
{\sigma_{\mathrm{i}-\mathrm{U}}}^{2}=k_{0}{\sigma_{\mathrm{n}}}^{2}\left(\left(x-x_{i}\right)^{2}+\left(y-y_{i}\right)^{2}+h^{2}\right),
\end{gathered}
$$

where $\sigma_{\mathrm{i}-\mathrm{VV}}^{2}, \sigma_{\mathrm{i}-\mathrm{HH}}^{2}, \sigma_{\mathrm{i}-\mathrm{VH}}^{2}$, and $\sigma_{\mathrm{i}-\mathrm{U}}^{2}$, are respectively the variance of the TOA measurement noise for the $\mathrm{VV}, \mathrm{HH}$, VH, and 'Uniform/Isotropic' antenna patterns at the $i^{\text {th }}$ sensor.

\section{Extraction of the TDOA from Measurements}

As discussed in Section II] the TDOA observations are obtained by computing the difference between two
TOA estimates. In this paper, we use a common reference sensor, with respect to which all the TDOA measurements are made. We choose $S-1$ in Fig. 1, as the reference sensor, and hence, with $N$ sensors, we obtain a TDOA measurement vector with $N-1$ elements. The true TDOA and its measured value are then given by $\tau_{1 i}=\frac{d_{1}}{c}-$ $\frac{d_{i}}{c}$ and $\quad \hat{\tau}_{1 i}=\hat{\tau}_{1}-\hat{\tau}_{i}$, respectively. Thus $\hat{\tau}_{1 i}$ is a Gaussian random variable $\hat{\tau}_{1 \mathrm{i}} \sim \mathcal{N}\left(\frac{d_{1}-d_{i}}{c}, \sigma_{1}^{2}+\sigma_{i}^{2}\right)$. Due to having a common reference sensor, the TDOA measurements are not independent, and the dependence is modeled through the covariance matrix $\boldsymbol{R}(\mathbf{x})$. Thus the TDOA measurement vector $\boldsymbol{z}=\left[\hat{\tau}_{12}, \hat{\tau}_{13} \ldots \hat{\tau}_{1 N}\right]$ is jointly-Gaussian distributed $\boldsymbol{z} \sim \mathcal{N}(\boldsymbol{\mu}(\mathbf{x}), \boldsymbol{R}(\mathbf{x}))$. The mean vector and the covariance matrix have been parameterized to show their dependence on the unknown location of the UAV, $\mathbf{x}=(x, y, h)$, and they can be respectively written as follows:

$$
\begin{aligned}
\boldsymbol{\mu}(\mathbf{x})= & \frac{1}{c}\left[\left(d_{1}-d_{2}\right),\left(d_{1}-d_{3}\right) \ldots\left(d_{1}-d_{N}\right)\right], \quad(15) \\
\boldsymbol{R}(\mathbf{x}) & =\left(\begin{array}{cccc}
\sigma_{1}^{2}+\sigma_{2}^{2} & \sigma_{1}^{2} & \vdots & \sigma_{1}^{2} \\
\sigma_{1}^{2} & \sigma_{1}^{2}+\sigma_{3}^{2} & \vdots & \sigma_{1}^{2} \\
\vdots & \vdots & \ddots & \vdots \\
\sigma_{1}^{2} & \sigma_{1}^{2} & \cdots & \sigma_{1}^{2}+\sigma_{(N-1)}^{2}
\end{array}\right)
\end{aligned}
$$

\section{Localization CRLB With Antenna EFFECTS}

Given (15) and (16), the likelihood function for the TDOA measurement vector, $z$ can be written as a function of the UAV location $(x, y, h)$ as follows:

$$
p(\boldsymbol{z} \mid \boldsymbol{x})=\frac{\exp \left(\frac{-(\boldsymbol{z}-\boldsymbol{\mu}(\boldsymbol{x})) \boldsymbol{R}^{-1}(\boldsymbol{x})(\boldsymbol{z}-\boldsymbol{\mu}(\boldsymbol{x}))^{T}}{2}\right)}{\sqrt{2 \pi}|\boldsymbol{R}(\boldsymbol{x})|},
$$

and therefore, the ML estimate of $\boldsymbol{x}$ is given by:

$$
\hat{\boldsymbol{x}}=\arg \max _{\boldsymbol{x}} p(\boldsymbol{z} \mid \boldsymbol{x}) .
$$

In our model both the mean $\boldsymbol{\mu}(\boldsymbol{x})$, and the covariance $\boldsymbol{R}(\boldsymbol{x})$ of the PDF is dependent on the unknown UE location $\boldsymbol{x}=(x, y, h)$. Therefore, the relationship between the covariance matrix $\boldsymbol{c}_{\hat{\boldsymbol{x}}}$ of an unbiased vector estimator $\hat{\boldsymbol{x}}$ and the Fisher information matrix (FIM), $I_{\hat{\boldsymbol{x}}}$ can be given as [12]:

$$
c_{\hat{\boldsymbol{x}}}-\boldsymbol{I}_{\hat{\boldsymbol{x}}}^{-1} \geq \mathbf{0}
$$

where, for $i=0,1,2$, and $j=0,1,2$, the $(i, j)$ element of the FIM is given as:

$$
\begin{aligned}
I_{i j} & =\frac{\partial \boldsymbol{\mu}(\boldsymbol{x})}{\partial x_{i}} \boldsymbol{R}^{-1}(\boldsymbol{x}) \frac{\partial \boldsymbol{\mu}(\boldsymbol{x})^{T}}{\partial x_{i}} \\
& +\frac{1}{2} \operatorname{trace}\left(\boldsymbol{R}^{-1}(\boldsymbol{x}) \frac{\partial \boldsymbol{R}(\boldsymbol{x})}{\partial x_{i}} \boldsymbol{R}^{-1}(\boldsymbol{x}) \frac{\partial \boldsymbol{R}(\boldsymbol{x})}{\partial x_{j}}\right),
\end{aligned}
$$

where,

$$
\begin{gathered}
\frac{\partial \boldsymbol{\mu}}{\partial x_{i}}(\mathbf{x})=\frac{1}{c}\left[\frac{\partial\left(d_{1}-d_{2}\right)}{\partial x_{i}}, \frac{\partial\left(d_{1}-d_{3}\right)}{\partial x_{i}} \cdots\right. \\
\frac{\partial \boldsymbol{R}(\mathbf{x})}{\partial x_{i}}=\left(\begin{array}{cccc}
\frac{\partial\left(\sigma_{1}^{2}+\sigma_{2}^{2}\right)}{\partial x_{i}} & \frac{\partial \sigma_{1}^{2}}{\partial x_{i}} & \cdots & \frac{\partial d_{1}^{2}}{\partial x_{i}} \\
\frac{\partial \sigma_{1}^{2}}{\partial x_{i}} & \frac{\partial\left(\sigma_{1}^{2}+\sigma_{3}^{2}\right)}{\partial x_{i}} & \cdots & \frac{\partial \sigma_{1}^{2}}{\partial x_{i}} \\
\vdots & \vdots & \ddots & \vdots \\
\frac{\partial \sigma_{1}^{2}}{\partial x_{i}} & \frac{\partial \sigma_{1}^{2}}{\partial x_{i}} & \cdots & \frac{\partial\left(\sigma_{1}^{2}+\sigma_{M}^{2}\right)}{\partial x_{i}}
\end{array}\right),
\end{gathered}
$$

with $M=(N-1)$. The partial derivatives in the above equations can be obtained using (11) through (13), and 
(15). Thus, the mean square error (MSE) can be given written as:

$$
E(x, y, h) \geq \operatorname{trace}\left[\boldsymbol{I}^{-1}(x, y, h)\right] .
$$

\section{LOCALIZATION METRICS}

In this section we introduce a number of localization performance metrics that we use for the evaluation of the localization performance in the rest of this paper. First of all, we define the system area under the consideration, as a subset of the $2 \mathrm{D}$ Euclidean plane, $\mathcal{A} \subset \mathbb{R}^{2}$. From the (21), we see that the CRLB-RMSE for a drone location $(x, y, h)$, is a function of the true value of the parameters. Then for a constant drone height of $h, E$ can be defined as a mapping from $\mathcal{A} \rightarrow \mathbb{R}$. The distribution of the random variable $E$, can then be written as

$$
F_{E}(\delta)=\operatorname{Pr}(E \leq \delta) \text {. }
$$

\section{A. Coverage Metrics}

For the purpose of this paper we use the Boolean notion of coverage, where the drone location $(x, y, h)$ is considered to be covered under the localization scheme, if the CRLB-RMSE at $(x, y, h)$ is less than a certain threshold $\delta$. Similarly, a sub-region of the system area, $\mathcal{A}_{i} \subset \mathcal{A}$, is considered to be covered under the localization scheme, if $\forall(x, y) \in \mathcal{A}_{i}$, the MSE, $E(x, y) \leq \delta$. Thus we define a coverage function as:

$$
\operatorname{Cov}\left(\mathcal{A}_{i}\right)= \begin{cases}1, & \text { if } \forall(x, y) \in \mathcal{A}_{i}, E(x, y) \leq \delta \\ 0, & \text { Otherwise }\end{cases}
$$

We then consider the following two coverage metrics.

a) Area Coverage Efficiency (ACE): The ACE is a measure of the aggregate localization coverage over the entire system area. We define ACE as the ratio of the area that is covered according to the Boolean notion of coverage to the entire system area. Thus the mathematical definition of ACE can be given as below:

$$
\operatorname{ACE}(\mathcal{A}, \delta, \zeta)=\frac{\operatorname{area}\left(\bigcup_{i: C o v}\left(\mathcal{A}_{i}\right)=1\right.}{\operatorname{area}(\mathcal{A})} .
$$

From the fundamental definition of probability, (24) can be expressed in terms of the distribution function of $E$, as below:

$$
\operatorname{ACE}(\mathcal{A}, \delta)=\operatorname{Pr}(E \leq \delta ; \zeta)=F_{E}(\delta, \zeta) .
$$

In the rest of this paper, we assume that the ACE is measured in conjunction with $\delta=100$ meters, and is named as the 100-meters-error-bound-coverage $\%$.

b) Quality of Localization ( $Q O L)$ : As we can see, if the error tolerance threshold, $\delta$ is increased, due to the non-decreasing nature of a cumulative distribution function (CDF), the value of the ACE will also be higher, while on the other hand the QOL will be diluted. Thus, we use the required error tolerance threshold, $\delta$ to achieve the targeted $p \%$ ACE, as a combined measure of the QOL and the ACE. The QOL can be mathematically defined as:

$$
\operatorname{QOL}(\mathcal{A}, p, \zeta)=F_{E}{ }^{-1}(p, \zeta) \text {. }
$$

In this paper, we use $p=80 \%$, as the required ACE against which we measure the QOL, and the metric is termed as the $80 \%$-coverage-error-bound.

\section{B. Accuracy Metrics}

In this paper, we use a most commonly used measure of accuracy, the median RMSE. The median RMSE is defined as below:

$$
\operatorname{Median}(E)=F_{E}^{-1}(0.5)
$$

As we can see the median is the same as the QOL, subject to $50 \%$ coverage. However, we don't use the median as a measure of the QOL, as a requirement of $\mathrm{ACE}=50 \%$, is too low for any practical application.

\section{NumericAl RESUlTS}

In this section, we present numerical results on the performance of the TDOA-based localization scheme for the four antenna patterns, using the CRLB expression derived in Section III. In future extension of this work, we intend to corroborate these analytical results with simulation results as well. We consider an area of size $1000 \times 1000 \mathrm{~m}^{2}$ with the origin located at the origin $(0,0) \mathrm{m}$, and $4 \mathrm{RF}$ sensors placed at $(250,250) \mathrm{m}$, $(-250,250) \mathrm{m},(-250,-250) \mathrm{m}$, and $(250,-250) \mathrm{m}$. We also assume that the RF sensors are operating at the carrier frequency of $5.8 \mathrm{GHz}$, and that the bandwidth and the transmit power of the drone are $10 \mathrm{MHz}$ and $20 \mathrm{dBm}$, respectively [4], [5], and that the noise power spectral density (PSD) is $-174 \mathrm{dBm} / \mathrm{Hz}$ [14].

In order to characterize the coverage performance provided by the different antenna patterns, we plot the CDFs in Fig. 3 for corresponding CRLB-RMSE. In each subplot of Fig. 3. we provide two horizontal lines (i.e., in magenta and black), which represent the $80 \%$ (i.e, $80 \%$-coverage-error-bound) and $50 \%$ (i.e., median) coverage based on RMSE with $F_{E}(e)=80 \%$ and $F_{E}(e)=50 \%$, respectively. In addition, the vertical line (i.e., in blue) represents the coverage based on $\delta=100$ (i.e., 100-meters-error-bound-coverage). From Fig. 3(a), we observe that at very low drone altitudes, such as 10 meters, the VH antenna pattern yields the lowest median RMSE, the lowest $80 \%$-coverage-error-bound, and the highest 100 -meters-error-bound-coverage $\%$. Thus the VH pattern provides the best localization performance in terms of both accuracy and area coverage, followed by the VV, and the $\mathrm{HH}$ pattern. We also observe that the $\mathrm{VV}$, and the 'Uniform' pattern have almost identical distributions of RMSE at very low altitudes.

Next, from Fig. 3. b), we observe that for medium drone altitudes of about 100 meters, the VV pattern yields the highest accuracy in terms of the median RMSE, and the highest quality of localization (QOL) in terms of the $80 \%$-coverage-error-bound. However we also note that the VV and the HH CDFs intersect above the horizontal line, $F_{E}(e)=0.9$, indicating that if we measured the QOL in conjunction with a higher requirement for ACE, such as $\operatorname{Pr}(E \leq \delta) \geq 90 \%$, then the $\mathrm{HH}$ pattern would provide a lower $90 \%$-coverage-error-bound and thus higher QOL as compared to the VV, and the 'Uniform' pattern. Fig. 3 (b) also shows us that, the value of the 100 -meters-error-bound-coverage $\%$ is the highest for the $\mathrm{HH}$ curve, i.e. the value of the ACE subject to an error tolerance of 100 meters is maximized for the $\mathrm{HH}$ antenna pattern.

On the other hand, if we considered the ACE metric to be subject to a lower error tolerance such as $\delta \leq$ 50 meters, then the maximum ACE will be obtained from the VV and the 'Uniform' pattern, followed by the VH pattern, instead of the HH pattern. From Fig. 3 (c), and (d), we observe that at very high drone altitudes, such as 300 to 500 meters, the lowest median RMSE, the lowest $80 \%$-coverage-error-bound, and the highest 100 -meters-error-bound-coverage $\%$ are achieved by the 'Uniform' pattern. While the performance of the VV pattern closely follows that of the 'Uniform' pattern, we 


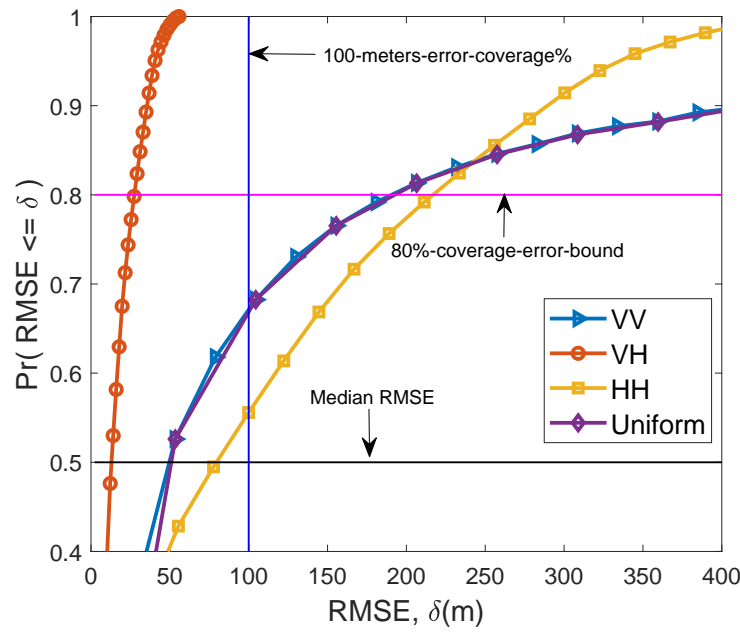

(a) Drone height: 10 meters.

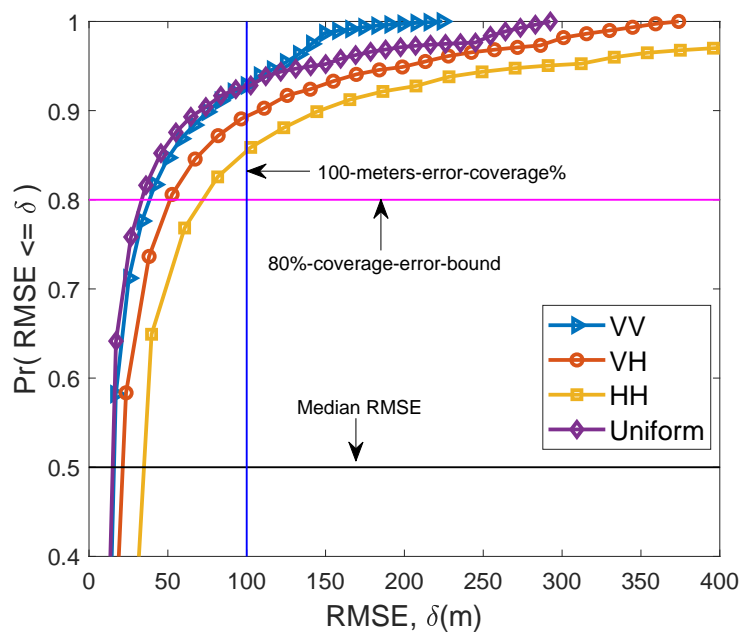

(c) Drone height: 300 meters.

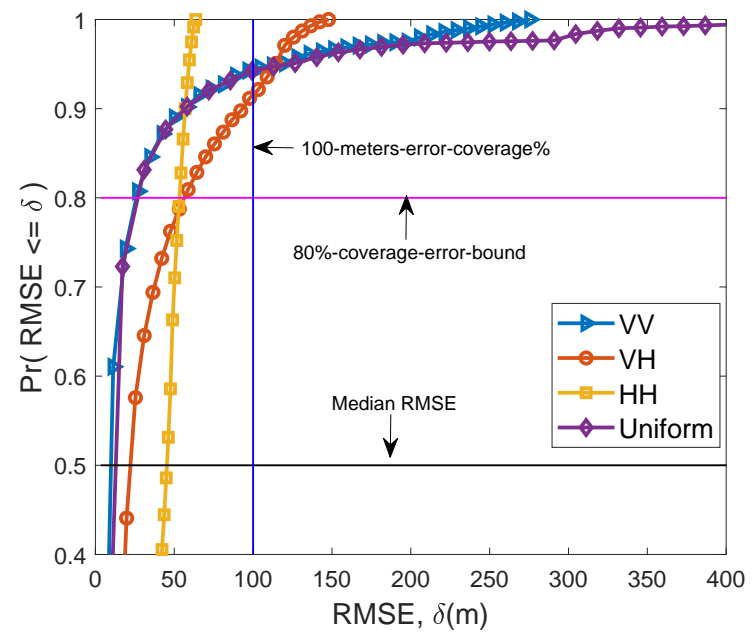

(b) Drone height: 100 meters.

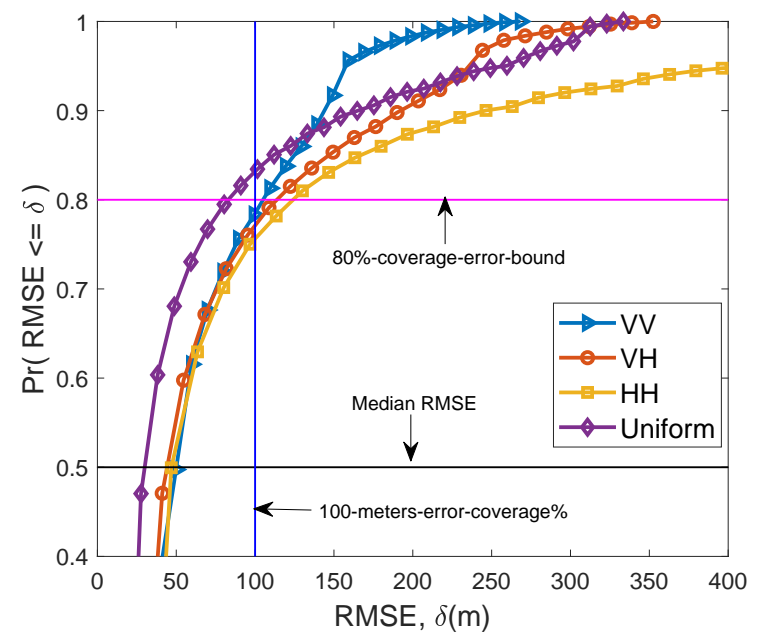

(d) Drone height: 500 meters.

Fig. 3: Cumulative distributions of CRLB RMSE for various antenna orientations (VV, VH, HH, Uniform) at UAV altitudes of (a) 10 meters. (b) 100 meters, (c) 300 meters, and (b) 500 meters.

note that in the case of a very high $\mathrm{ACE}$ requirement $(\operatorname{Pr}(E \leq \delta) \geq 90 \%)$, the the highest QOL is achieved by the $\mathrm{VV}$ pattern, and in the case of a higher error tolerance ( $\delta \geq 150$ meters), the higher ACE is also provided by the VV pattern. Unlike, low and medium altitudes, at very high altitudes, we see more differentiation between the RMSE distributions for the VV and the 'Uniform' pattern, and the CDFs of the $\mathrm{VH}$ and the $\mathrm{HH}$ patterns move closer to that of the 'Uniform' pattern. This can be explained by the fact that at lower altitudes the elevation angle is very small, thus the cosine of the elevation angle, and the antenna gain for the VV pattern is very close to the gain for the the 'Uniform' pattern, 1.

As the altitude increases, the elevation angle becomes larger, resulting in lower antenna gain for the VV pattern, and higher antenna gain for the $\mathrm{VH}$, and the $\mathrm{HH}$ pattern. Therefore the performance gap between the VV and the 'Uniform' pattern becomes larger, while the performance gap between the HH/VH pattern and the 'Uniform' pattern becomes smaller.

Having realized the importance of characterizing the localization performance with respect to the drone altitude, we plot these performance metrics as a function of the drone altitude, in Fig. 4, Fig. 5, and Fig. 6. From these plots, we observe a general pattern, that is, at very low altitudes below 50 meters, all

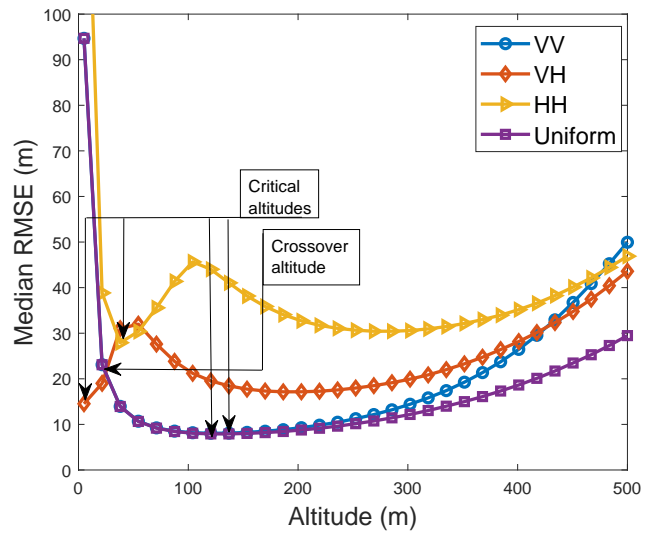

Fig. 4: Median RMSE as a function of drone altitude for various antenna patterns using 4 sensors.

antenna patterns other than the $\mathrm{VH}$ pattern, yield very high errors, therefore we observe very high median RMSE, and $80 \%$-coverage-error-bound, and very low 100 -meters-error-bound-coverage $\%$. Beyond 50 meters, the performance for these antenna patterns start to improve, and reaches the optimal point at a certain altitudes, but again starts to degrade at very high altitudes. Thus we realize, that there is a critical height where the performance metrics are optimized. For the VH pattern, 


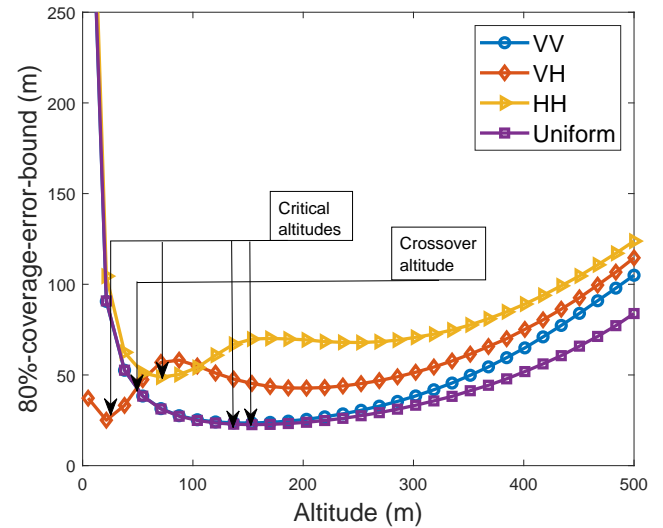

Fig. 5: 80\%-coverage-error-bound as a function of drone altitude for various antenna patterns using 4 sensors.

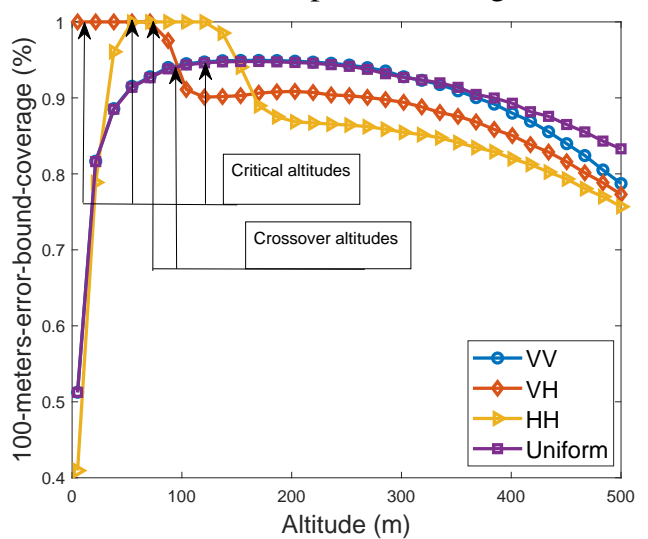

Fig. 6: 100-meters-error-bound-coverage $\%$ as a function of drone altitude for various antenna patterns using 4 sensors.

the performance is optimized at very low altitudes such as below 50 meters. The local maximas/minimas for the different metrics, have been annotated in the corresponding plots for all the antenna patterns. Table $\mathrm{II}$ summarizes the results.

The points of intersection between the different antenna pattern curves, in Fig. 4, Fig. 5, and Fig. 6, indicate that the choice of the best antenna pattern with respect to the given performance metric, changes before and after the corresponding point of intersection. The abscissa of the point of intersection gives us the crossover height, beyond which the choice of the best antenna pattern changes. These crossover altitudes have been annotated in the plots. We observe that, in terms of the median RMSE the crossover altitude is 21.5 meters. Below 21.5 meters the highest accuracy is achieved by the VH pattern, and beoynd 21.5 meters the accuracy is maximized by the 'Uniform' pattern. In terms of QOL, the lowest ept is provided by the $\mathrm{VH}$ pattern for drone altitudes below 55 meters, beyond which the best QOL is achieved by the 'Uniform' pattern. Lastly we note that, when ACE is measured in terms of the hundc, the best coverage performance is provided the $\mathrm{VH}$ pattern till the drone altitude of 71 meters, whereas between 71 meters and 121 meters, the HH pattern, and beyond 121 meters, the 'Uniform' pattern, respectively, yield the highest ACE.

\section{CONCLUSION}

In this paper a TDOA-based RF positioning system for localization of drones is studied in conjunction with a simple air-to-ground 3D antenna radiation pattern. Our results show that accounting for antenna effects
TABLE II: Critical drone altitude with respect to various performance metrics for 4 sensors.

\begin{tabular}{|c||c|c|c|c|}
\hline & VV & VH & HH & Uniform \\
\hline Median RMSE & 130 & 5 & 15 & 45 \\
\hline $80 \%$-coverage-error-bound & 145 & 20 & 75 & 155 \\
\hline 100 -meters-error-bound-coverage $\%$ & 160 & 5 & 55 & 150 \\
\hline
\end{tabular}

makes a significant difference and reveals many important relationships between the localization accuracy and the altitude of the drone. It is seen that the localization performance varies in a non-monotonic pattern with respect to the drone altitude. We are also able to characterize, the critical heights, where the coverage and accuracy metrics are optimized, and the crossover UAV heights, before and after which, the choice of the best antenna pattern yielding best performance changes. In the future, we intend to extend this work, primarily by carrying out a similar analysis on the localization CRLB in conjunction with a more realistic antenna pattern, and then finding the optimal placement of the ground sensors, that minimizes the localization CRLB.

\section{REFERENCES}

[1] Y. Zeng, R. Zhang, and T. J. Lim, "Wireless communications with unmanned aerial vehicles: opportunities and challenges," IEEE Commun. Mag., vol. 54, no. 5, pp. 36-42, May 2016.

[2] I. Guvenc, F. Koohifar, S. Singh, M. L. Sichitiu, and D. Matolak, "Detection, tracking, and interdiction for amateur drones," IEEE Commun. Mag., vol. 56, no. 4, pp. 75-81, 2018.

[3] M. M. Azari, H. Sallouha, A. Chiumento, S. Rajendran, E. Vinogradov, and S. Pollin, "Key technologies and system trade-offs for detection and localization of amateur drones," IEEE Commun. Mag., vol. 56, no. 1, pp. 51-57, 2018.

[4] K. Chang, "RF sensor for interference detection-an RF signal-based drone detection system," KeySight Technologies, 2016.

[5] T. Boon-Poh, "RF techniques for detection, classification and location of commercial drone controllers," KeySight Technologies, Aerospace Defense Symposium, 2017.

[6] S. Gezici, "A survey on wireless position estimation," Wirel. Pers. Commun., vol. 44, no. 3, pp. 263-282, Feb. 2008.

[7] I. Guvenc and C. Chong, "A survey on TOA based wireles localization and NLOS mitigation techniques," IEEE Commun. Surveys Tutorials, vol. 11, no. 3, pp. 107-124, rd 2009.

[8] N. Patwari, A. O. Hero, M. Perkins, N. S. Correal, and R. J. O'Dea, "Relative location estimation in wireless sensor networks," IEEE Trans. Sig. Proc., vol. 51, no. 8, pp. 2137-2148, Aug 2003.

[9] N. Vankayalapati, S. Kay, and Q. Ding, "TDOA based direct positioning maximum likelihood estimator and the Cramer-Rao bound," IEEE Trans. Aerospace Electronic Syst., vol. 50, no. 3, pp. 1616-1635, July 2014.

[10] J. Chen, D. Raye, W. Khawaja, P. Sinha, and I. Guvenc, "Impact of 3D UWB Antenna Radiation Pattern on Air-to-Ground Drone Connectivity Jianlin Chen,", in Proc. IEEE Vehic. Technol. Conf. (VTC), Chicago, IL, Sep. 2018.

[11] W. Khwaja, I. Guvenc, D. Matolak, U.-C. Fiebig, and Schneckenberger, "A survey of air-to-ground propagation channe; modeling for unmanned aerial vehicles," available online arxiv.org/abs/1801.01656, 2018

[12] S. M. Kay, Fundamentals of Statistical Signal Processing: Estimation Theory. Upper Saddle River, NJ, USA: Prentice-Hall, Inc., 1993.

[13] R. Zekavat and R. M. Buehrer, Handbook of Position Location: Theory, Practice and Advances, 1st ed. Wiley-IEEE Press, 2011.

[14] A. Carlson, P. Crilly, and J. Rutledge, Communication Systems: An Introduction to Signals and Noise in Electrical Communication. McGraw-Hill, 2002. 